\title{
Mortalidade de mulheres com câncer do colo do útero: revisão integrativa da literatura
}

\author{
Mortality of women with cervical cancer: integrative literature review \\ Mortalidad de mujeres con cáncer cervical: revisión integradora de la literatura
}

\begin{abstract}
RESUMO
Objetivo: identificar produções científicas que apresentem dados significativos sobre mortalidade e sobrevida de mulheres com câncer do colo do útero. Método: revisão integrativa da literatura, realizada através de bases de dados Scientific Electronic Library Online (SciELO) e Literatura Latino-americana e do Caribe em Ciências da Saúde (LILACS), mediante a utilização de Descritores em Ciências da Saúde (DECS), abrangendo artigos publicados no período de 2008 a 2018. Resultados: foram incluídos 13 artigos ao estudo. Ao término dos resultados foram agrupados as seguintes categorias: Fatores associados a mortalidade por câncer do colo do útero; Mortalidade e sobrevida por CCU no Brasil; Ações para redução da mortalidade pelo câncer do colo do útero. Conclusão: o Câncer de Colo do Útero é considerado um problema de saúde pública, refletindo diretamente nos indicadores de morbimortalidade do País, variando de região para região e estando diretamente associado a oferta de serviços de rastreamento, diagnóstico e tratamento.
\end{abstract}

DESCRITORES: Mortalidade; Sobrevida; Câncer de Colo do Útero; Saúde da Mulher.

\section{ABSTRACT}

Objective: to identify scientific productions that present significant data on mortality and survival of women with cervical cancer. Method: integrative literature review, carried out through Scientific Electronic Library Online (SciELO) and Latin American and Caribbean Literature in Health Sciences (LILACS) databases, using Health Sciences Descriptors (DECS), covering articles published from 2008 to 2018. Results: 13 articles were included in the study. At the end of the results, the following categories were grouped: Factors associated with mortality from cervical cancer; Mortality and survival due to CCU in Brazil; Actions to reduce mortality from cervical cancer. Conclusion: Cervical Cancer is considered a public health problem, directly reflecting on the country's morbidity and mortality indicators, varying from region to region and being directly associated with the provision of screening, diagnosis and treatment services.

DESCRIPTORS: Mortality; Survival; Cervical Cancer; Women's Health.

\section{RESUMEN}

Objetivo: identificar producciones científicas que presenten datos significativos sobre mortalidad y supervivencia de mujeres con cáncer de cuello uterino. Método: revisión integrativa de la literatura, realizada a través de las bases de datos Scientific Electronic Library Online (SciELO) y Literatura Latinoamericana y del Caribe en Ciencias de la Salud (LILACS), utilizando Descriptores de Ciencias de la Salud (DECS), cubriendo artículos publicados de 2008 a 2018. Resultados: 13 artículos fueron incluidos en el estudio. Al final de los resultados, se agruparon las siguientes categorías: Factores asociados a la mortalidad por cáncer de cuello uterino; Mortalidad y supervivencia por UCC en Brasil; Acciones para reducir la mortalidad por cáncer cervicouterino. Conclusión: el cáncer cervicouterino se considera un problema de salud pública, que refleja directamente los indicadores de morbilidad y mortalidad del país, varía de región a región y está directamente asociado con la prestación de servicios de tamizaje, diagnóstico y tratamiento.

DESCRIPTORES: Mortalidad; Supervivencia; Cáncer de cuello uterino; La salud de la mujer.

RECEIVED: 12/10/2021 APPROVED : 15/11/2021

Allana Raquel Monteiro Assunção

Enfermeira Faculdade de Saúde, Ciências Humanas e Tecnológicas do Piauí - NOVAFAPI.. Faculdade de Saúde, Ciências Humanas e Tecnológicas do Piauí - NOVAFAPI.

ORCID:

Francilene de Sousa Vieira

Enfermeira. Mestre em Biodiversidade, Ambiente e Saúde (UEMA). Universidade Federal do Ceará

ORCID: 0000-0003-3099-6032 


\section{Gleciane Costa de Sousa}

Enfermeira. Mestre em Biodiversidade, Ambiente e Saúde (UEMA). Universidade Estadual do Maranhão (UEMA)

ORCID: 0000-0001-9745-7645

\section{Julianne de Area Leão Pereira da Silva}

Enfermeira Faculdade de Saúde, Ciências Humanas e Tecnológicas do Piauí - NOVAFAPI.. Faculdade de Saúde, Ciências Humanas e Tecnológicas do Piauí - NOVAFAPI.

ORCID: 0000-0003-4529-0104

\section{Gizelia Araújo Cunha Porto}

Enfermeira. Mestre em Biodiversidade, Ambiente e Saúde (UEMA). Universidade Estadual do Maranhão.

ORCID: 0000-0001-5363-4646

\section{Shirley Dayane Santana da Silva Almones}

Faculdade de Ciências e Tecnologia do Maranhão (FACEMA). Enfermeira.Especialista em Urgência, Emergência e Atendimento Pré-hospitalar.

\section{INTRODUÇÃO}

O câncer é uma das doenças mais temidas no mundo, grande parte se deve a ausência de tratamento efetivo para a maioria dos tumores metastáticos. No entanto nos últimos 40 anos houve uma progressão no tratamento cirúrgico, radioterapia e na quimioterapia tendo como consequência a elevação na expectativa de vidal.

$\mathrm{O}$ câncer pode ser subdividido em três fases, principais, que esclarecem os eventos cronológicos sequenciais da carcinogênese, são eles: iniciação e promoção (que começa na fase latente) e progressão (que começa na fase clínica) 1 .

$\mathrm{O}$ câncer de colo uterino (CCU) também conhecido como câncer de cérvice uterina é a neoplasia genital mais frequente na mulher brasileira, excetuando-se os casos de câncer de pele não melanoma. Caracteriza-se por ser normalmente uma doença de evolução lenta que acomete, sobretudo, mulheres acima de 25 anos.

Segundo dados obtidos no Instituto $\mathrm{Na}$ cional do Câncer (INCA), o câncer de colo uterino representa o segundo tipo de câncer mais comum entre as mulheres, com aproximadamente 500 mil casos novos por ano no mundo, responsável pelo óbito de, aproximadamente, 230 mil mulheres por ano2.

De acordo com o Ministério da Saúde se desconsiderarmos, os tumores da pele não melanoma, o câncer do colo do útero é o mais incidente, na região Norte (1.360/100 mil). Já nas regiões Centro-Oeste (2.020/100 mil) e Nordeste (5.050/100 mil) ocupam a segunda posição mais frequente, na região Sudeste $(8.610 / 100 \mathrm{mil})$, a terceira, e na região Sul (2.000/100 mil), a quarta posição. A análise dos dados nos permite verificar que a distribuição dos dados é heterogênea2.

O controle desta neoplasia acompanha a estratégia de prevenção secundária embasada na citologia cervical. Este método de detecção, conhecido popularmente como Papanicolau ou exame preventivo, vem sendo realizado por mais de 30 anos. Apesar de o exame preventivo ser extremamente simples, inofensivo, eficiente, de baixo custo, o câncer cérvico-uterino ainda possui uma elevada incidência entre as mulheres brasileiras3.

Segundo estudos realizados, o principal fator de risco para o desenvolvimento de lesões intraepiteliais de alto grau e do câncer do colo do útero é a infecção pelo HPV. Embora o HPV seja uma condição necessária para existência deste tipo de câncer, não é uma condição suficiente para existir câncer de colo do útero sem a existência de outros fatores predisponentes 4 .

Baseada na problemática que envolve o câncer de cérvice-uterino, mostra-se relevante a realização de uma pesquisa que tem como objeto de estudo conhecer e analisar os dados de mortalidade e sobrevida dessa condição clínica.

\section{MÉTODOS}

Este trabalho configura-se como uma revisão do tipo integrativa. Este tipo de revisão consiste na coleta de informações em banco de dados, permitindo dessa forma a obtenção de conclusões oriundas de uma questão norteadora. No processo de elaboração da revisão integrativa, esta deve ser realizada através de métodos rigorosos, apresentando clareza e consistência nos métodos utilizados para extração das informações dos estudos primários5.

A coleta de dados foi realizada através de bases de dados Scientific Electronic Library Online - SciELO, e Literatura Latino-americana e do Caribe em Ciências da Saúde - LILACS, mediante a utilização de Descritores em Ciências da Saúde (DeCS), abrangendo artigos publicados no período de 2008 a 2018.

A seleção dos estudos para análise obedeceu aos seguintes critérios de inclusão: 1) Abordagem da temática investigada, 2) Em forma de artigo, 3) Idioma português, 4) Publicados no período de 2008 a 2018, 5) Texto completo disponível. Os critérios de exclusão foram: 1) Artigo não disponível, 2) Repetição de um mesmo artigo em mais de um banco de dados, 3) Não corresponder ao tema da pesquisa, 4) Artigo de revisão.

A partir da combinação dos descritores, aplicação dos critérios de exclusão e inclusão foram selecionados 54 artigos. Após lei- 
tura analítica dos trabalhos, apenas 13 artigos atendiam a todos os requisitos, fazendo parte desta pesquisa.

Para realização da análise dos trabalhos selecionados procedeu-se a divisão por etapas seguindo as recomendações de Souza, Silva e Carvalho6. A pesquisa foi operacionalizado através de etapas sistematizadas e interrelacionadas, consistindo inicialmente na elaboração da pergunta norteadora, investigação na literatura, obtenção de dados, análise crítica dos estudos incluídos, discussão dos resultados e apresentação da revisão integrativa.

A avaliação dos artigos teve como base os passos da realização de avaliação crítica de literatura do JAMA (Journal Of Ameri- can Medical Association) que são: 1) identificação e seleção dos estudos; 2) leitura crítica; 3) resumo do artigo; 4) apresentação; 5) perguntas; 6) síntese7. Para análise das informações foi realizada a organização do conteúdo encontrado quanto ao título, ano, abordagem metodológica, e objetivos do estudo.

\section{RESULTADOS}

O Quadro 01 apresenta a disposição dos artigos, segundo dados dos autores, título da publicação, períodico, ano e objetivo do trabalho permitindo a visualização dos trabalhos que fazem parte dessa revisão.

\section{DISCUSSÃO}

Fatores associados a mortalidade por câncer do colo do útero

O CCU encontra-se associado a inúmeros fatores, dentre eles destacam-se a forte associação entre idade e risco de câncer, recomendando a necessidade de se analisar o comportamento da mor $\neg$ talidade por câncer em diferentes grupos etários da população feminina, sob diferentes perspectivas, destacando ainda que, pesquisas com essa finalidade são limitados no Brasil, sendo dificultoso o conhecimento de sua distribuição na população feminina e suas possíveis relações com as tendências de mortalidade8.

\section{Quadro 01. Artigos selecionados para revisão compreendidos no período de 2008 a 2018 (BRASIL, 2018).t}

\begin{tabular}{|c|c|c|c|c|}
\hline AUTORES & TÍTULO & PERIÓDICO & $\begin{array}{c}\text { ANO DE } \\
\text { PUBLICAÇÃO }\end{array}$ & OBJETIVO \\
\hline $\begin{array}{l}\text { Neves, Silva, } \\
\text { Ribeiro et } \\
\text { al., }\end{array}$ & $\begin{array}{l}\text { Adversidades encontradas pelo enfer- } \\
\text { meiro para a realização da prevenção } \\
\text { do câncer do colo de útero }\end{array}$ & $\begin{array}{l}\text { Rev. Saúde } \\
\text { Coletiva }\end{array}$ & 2019 & $\begin{array}{l}\text { Avaliar as adversidades encontradas pelo enfer- } \\
\text { meiro para realização da prevenção do câncer } \\
\text { do colo de útero. }\end{array}$ \\
\hline $\begin{array}{l}\text { Barbosa, } \\
\text { Sousa, } \\
\text { Bernal et al., }\end{array}$ & $\begin{array}{c}\text { Desigualdades regionais na } \\
\text { mortalidade por câncer de colo de útero } \\
\text { no Brasil:tendências e projeções até o } \\
\text { ano } 2030\end{array}$ & $\begin{array}{l}\text { Ciência } \\
\text { \& Saúde } \\
\text { Coletiva }\end{array}$ & 2016 & $\begin{array}{l}\text { Analisar a tendência temporal da mortalidade } \\
\text { por cancer de colo de útero no Brasil e calcular } \\
\text { uma projeção até o ano de } 2030 \text {. }\end{array}$ \\
\hline $\begin{array}{l}\text { Meira; Silva; } \\
\text { Silva et al., }\end{array}$ & $\begin{array}{l}\text { Efeito idade-período-coorte na mortali- } \\
\text { dade por câncer do colo uterino }\end{array}$ & $\begin{array}{l}\text { Rev. Saúde } \\
\text { Pública }\end{array}$ & 2013 & $\begin{array}{l}\text { Estimar o efeito da idade, período e coorte de } \\
\text { nascimento na mortalidade por câncer do colo } \\
\text { do útero. }\end{array}$ \\
\hline $\begin{array}{l}\text { Gonzaga, } \\
\text { Freitas } \\
\text { Júnior, } \\
\text { Barbaresca } \\
\text { et al., }\end{array}$ & $\begin{array}{l}\text { Tendências de mortalidade por câncer } \\
\text { do colo do útero no Brasil: 1980-2009 }\end{array}$ & $\begin{array}{l}\text { Cad. Saúde } \\
\text { Pública }\end{array}$ & 2014 & $\begin{array}{l}\text { Descrever as tendências temporais nas taxas de } \\
\text { mortalidade por câncer do colo do útero no Bra- } \\
\text { sil e nas principais regiões geográficas e estados } \\
\text { do país de } 1980 \text { a } 2009 \text {. }\end{array}$ \\
\hline $\begin{array}{l}\text { Nakagawa, } \\
\text { Espinosa, } \\
\text { Barbieri et } \\
\text { al., }\end{array}$ & $\begin{array}{l}\text { Carcinoma do colo do útero: taxa de so- } \\
\text { brevida e fatores prognósticos em mu- } \\
\text { Iheres no Estado de Mato Grosso }\end{array}$ & $\begin{array}{l}\text { Acta. Paul. } \\
\text { Enferm. }\end{array}$ & 2011 & $\begin{array}{l}\text { Analisar a taxa de sobrevida de mulheres sub- } \\
\text { metidas ao tratamento de câncer do colo do } \\
\text { útero no Estado de Mato Grosso. }\end{array}$ \\
\hline $\begin{array}{l}\text { Santos, } \\
\text { Melo }\end{array}$ & $\begin{array}{c}\text { Mortalidade e assistência oncológica no } \\
\text { Rio de Janeiro: Câncer de mama e colo } \\
\text { uterino }\end{array}$ & $\begin{array}{l}\text { Esc. Anna } \\
\text { Nery }\end{array}$ & 2011 & $\begin{array}{c}\text { Analisar a trajetória dos óbitos por câncer de } \\
\text { mama e colo uterino no município do Rio de Ja- } \\
\text { neiro. }\end{array}$ \\
\hline $\begin{array}{l}\text { Bottero, } \\
\text { Cervantes, } \\
\text { Becerra }\end{array}$ & $\begin{array}{l}\text { Diferenças socioeconômicas nos anos } \\
\text { de vida perdidos por câncer de mama } \\
\text { e câncer cervical na Colômbia, } 1997 \text { e } \\
\qquad 2007\end{array}$ & $\begin{array}{l}\text { Rev. Gerenc. } \\
\text { Polit. Saúde }\end{array}$ & 2011 & $\begin{array}{l}\text { Contribuir para o conhecimento do efeito da } \\
\text { mortalidade por cancer cervical na Colômbia, de } \\
\text { acordo com os grupos etários e status sócio-e- } \\
\text { conômico para os anos de } 1997 \text { e } 2007 .\end{array}$ \\
\hline
\end{tabular}




\section{artigo}

Assunção, A. R. M.,Vieira, F. S., Sousa, G. C., Silva, J. A. L. P., Porto, G. A. C.,Almones, S. D. S. S

Mortality of women with cervical cancer: integrative literature review

\begin{tabular}{|c|c|c|c|c|}
\hline Carmo, Luiz & $\begin{array}{l}\text { Sobrevivência de uma coorte de mulhe- } \\
\text { res com câncer de colo uterino diagnos- } \\
\text { ticadas em um centro de câncer brasi- } \\
\text { leiro }\end{array}$ & $\begin{array}{l}\text { Rev. Saúde } \\
\text { Pública }\end{array}$ & 2011 & $\begin{array}{l}\text { Avaliar a sobrevida global de mulheres com cân- } \\
\text { cer de colo uterino. }\end{array}$ \\
\hline $\begin{array}{l}\text { Gamarra; } \\
\text { Valente; } \\
\text { Silva }\end{array}$ & $\begin{array}{c}\text { Magnitude da mortalidade por câncer } \\
\text { do colo do útero na Região Nordeste do } \\
\text { Brasil e fatores socioeconômicos }\end{array}$ & $\begin{array}{l}\text { Rev. Panam. } \\
\text { Salud Pu- } \\
\text { blica }\end{array}$ & 2010 & $\begin{array}{l}\text { Analisar a tendência temporal da mortalidade } \\
\text { por câncer do colo do útero em mulheres com } \\
20 \text { anos ou mais residentes no Nordeste do } \\
\text { Brasil (capital e interior) no período de } 1996 \text { a } \\
2005 \text {. }\end{array}$ \\
\hline $\begin{array}{l}\text { Silva, } \\
\text { Girianelli, } \\
\text { Gamarra et } \\
\text { al., }\end{array}$ & $\begin{array}{l}\text { Tendências de mortalidade por câncer } \\
\text { do colo do útero no Brasil, 1981-2006 }\end{array}$ & $\begin{array}{l}\text { Cad. Saúde } \\
\text { Pública }\end{array}$ & 2010 & $\begin{array}{l}\text { Descrever as tendências da mortalidade por } \\
\text { câncer do colo do útero no Brasil para o período } \\
\text { de 1981-2006. }\end{array}$ \\
\hline $\begin{array}{l}\text { Alves; Guer- } \\
\text { ra; Bastos }\end{array}$ & $\begin{array}{c}\text { Tendência de mortalidade por câncer de } \\
\text { colo de útero para o Estado de Minas } \\
\text { Gerais, Brasil, 1980-2005 }\end{array}$ & $\begin{array}{l}\text { Cad. Saúde } \\
\text { Pública }\end{array}$ & 2009 & $\begin{array}{c}\text { Avaliar a tendência da mortalidade por câncer } \\
\text { cervical no Estado de Minas Gerais, Brasil, no } \\
\text { período compreendido entre 1980-2005. }\end{array}$ \\
\hline $\begin{array}{l}\text { Basilio, } \\
\text { Mattos }\end{array}$ & $\begin{array}{l}\text { Câncer em mulheres idosas das regiões } \\
\text { Sul e Sudeste do Brasil: Evolução da } \\
\text { mortalidade no período } 1980 \text { - } 2005\end{array}$ & $\begin{array}{l}\text { Rev. Bras. } \\
\text { Epidemiol. }\end{array}$ & 2008 & $\begin{array}{l}\text { Analisar a tendência da mortalidade por loca- } \\
\text { lizações selecionadas de câncer na população } \\
\text { feminina de } 60 \text { anos ou mais das regiões Sul e } \\
\text { Sudeste do Brasil, no período 1980-2005. }\end{array}$ \\
\hline Zefferino & $\begin{array}{l}\text { O desafio de reduzir a mortalidade por } \\
\text { câncer do colo do útero }\end{array}$ & $\begin{array}{l}\text { Rev. Bras. } \\
\text { Ginecol. } \\
\text { Obstet. }\end{array}$ & 2008 & $\begin{array}{l}\text { Relatar o desafio de reduzir a mortalidade por } \\
\text { câncer do colo do útero. }\end{array}$ \\
\hline
\end{tabular}

Estudo ao observar pelo período de 60 meses a taxa de sobrevida global, esta correspondeu a $66,7 \%$, sendo o tempo médio de sobrevida de 23,5 meses, e o tempo mediano de sobrevida foi de 18,5 meses. A taxa de sobrevida global, em 60 meses, foi de $63,6 \%$ nas mulheres com idade inferior a 50 anos e de $71,4 \%$ aquelas na faixa etária dos 50 anos ou mais 9 .

Estudo apontou que o tipo histológico mais prevalente de tumor foi o carcinoma de células escamosas $(84,5 \%)$. A doença localmente avançada, estágios II e III foi observada em $68,3 \%$ da coorte. A maioria dos casos foi acompanhada por pelo menos dois anos $(80,7 \%)$ e foram registrados 1.499 óbitos (50,1\% dos casos). As mortes ocorreram com mais frequência nos dois primeiros anos de acompanhamento $(71,2 \%)$ e nos estádios II ou III, correspondendo a $84,9 \%$ de todas as mortes 10 .

As mulheres com ocupação remunerada apresentaram taxa de sobrevida de $70 \%$ e as sem ocupação remunerada, donas de casa, apresentaram taxa de sobrevida menor, de $65,9 \%$. Quanto às variáveis clínicas, observou-se que as mulheres que apresentaram queixas ou sintomas sugestivos da doença, tiveram um prognóstico pior, apresentando taxa de sobrevida de $53,1 \%$, e aquelas sem sintomas ou queixas tiveram taxa de sobrevida de $86,4 \%$, cuja diferença foi estatisticamente significativa9.

Nessa perspectiva, é essencial que as mulheres de 50 anos de idade submetidos à mamografia a cada dois anos, ainda que não apresente sintomatologia visível de acordo com a norma técnica, entretanto, não para desqualificar o papel potencial de autoexploração desde tenra idade, para o qual é essencial que as mulheres sejam educadas adequadamente sobre a técnical 1.

\section{Mortalidade e sobrevida por CCU no Brasil}

No Brasil, no período referente a 1996 e 2005, os valores das taxas anuais médias de óbitos por CCU correspondeu a 4,6 e 5,1 óbitos por 100.000 mulheres-ano. Os valores referentes às taxas de mortalidade apresentaram incrementos de 103,4\% no Brasil, ocorrendo variação de $35 \%$ para as capitais da região Sul e até 339\% para o interior da região Nordeste12.

A avaliação do risco de morte por câncer do colo do útero segundo as coortes de nascimento mostrou que as mulheres nascidas entre 1900-1920 apresentaram risco elevado de morte por essa neoplasia. As coortes de nascimento a partir de 1930 tiveram redução progressiva no risco de morte, verificando-se efeito de proteção $(R R<1)$ nas mulheres nascidas após 196013.

Análise da tendência de mortalidade no Brasil mostrou a ocorrência de estabilização, houve nas principais regiōes geográficas uma tendência de queda no Sul a partir de 1999, Sudeste de 2001 a 2009, e Centro-Oeste, no entanto a região Nordeste e Norte mostraram uma tendência ascendente na mortalidade, enquanto o sul do Brasil mostrou uma tendência de queda em todos as unidades de federação a partir do ano 200014.

De 1996 a 2010, foram registrados no Brasil 89.764 óbitos por neoplasia maligna de colo de útero. A taxa de mortalidade padronizada à população mundial para o Brasil variou de 8,04 óbitos por 100.000 habitantes, no ano de 1996 a 6,36 óbitos 
por 100.000 habitantes, no ano de 201015.

A organização, integralidade e qualidade do programa de rastreamento através do teste de Papanicolau, o seguimento das pacientes, contribui significativamente para a redução da mortalidade específica, pois a oferta e qualidade dos serviços de saúde prestados exercem influência direta na sobrevida dos pacientes 16 .

Dessa forma, ressalta-se a importância da implantação e consolidação do Programa Nacional de Controle do Câncer do Colo do útero (PNCCC) na redução do risco de morte por CCU, inclusive nas mulheres com maior exposição aos fatores de risco para essa neoplasia13.

É importante ressaltar que as tendências de mortalidade por câncer do colo do útero mostraram um padrão diferencial de acordo com as regióes geográficas do país, refletindo indiretamente uma relação com o perfil socioeconômico das populações residentes nessas regiões. Entre os municípios do interior, houve uma tendência de alta no Norte e Nordeste, uma tendência de queda no Sul e Sudeste, e uma tendência estável no Centro-Oeste17.

\section{Ações para redução da mortalidade pelo câncer do colo do útero}

O Câncer em pauta neste trabalho, quando da ocorrência em países desenvolvidos que dispóe em seu arcabouço tecnologias de ponto para o diagnóstico e tratamento, tem limitado a efetividade do tratamento, visto que os dados relativos às taxas de sobrevivência não tem ultrapassado os valores de 70\% a 75\%. Dessa forma, entende-se que, para o combate à doença, se faz necessário intervir nas fases iniciais, utilizando medidas de precaução e diagnóstico precoce, com menor custo possível, de forma a tornar um recurso acessível a todas as camadas populares, dando ênfase à educação e ao autocuidado da população feminina 9.

Estudo mostrou que as mulheres apenas se sentem motivadas para a realização do exame preventivo apenas quando existe a presença de sinais e sintomas capazes de causar algum incômodo. Isso demonstra que os indivíduos procuram o serviço de saúde apenas quando a doença já encontra-se instalada no organismo o que impossibilita o diagnóstico precoce e prevenção da doença18.

A realização de exames de rastreamento, no entanto não é medida suficiente para garantir a redução da mortalidade. Faz-se necessário o tratamento adequado diante da detecção de alterações. O eficiente controle do CCU está intimamente diretamente relacionado com a qualidade do sistema de saúde, e além disso fornecer um diagnóstico e tratamento preciso; acesso fácil e ágil aos serviços; flexibilidade para marcação e remarcação de consultas e agilidade no atendimento19.
Finalmente, cabe destacar que a educação em saúde, o aprimoramento dos programas de rastreamento, a melhoria da qualidade da informação e, até mesmo, o aumento da abrangência dos RCBP devem ser considerados como importantes estratégias a serem adotadas para a redução da mortalidade e das repercussões físicas, psíquicas e sociais do câncer de colo uterino em nosso país 20 .

\section{CONCLUSÃO}

O Câncer do Colo do Útero configura-se como um problema de saúde pública, que tem impacto direto sobre os indicadores de morbidade e mortalidade, acometendo mulheres em idades variadas, onde a literatura aponta que mulheres em idade inferior a 60 anos, em grau avançada da doença, em que ocorre metástase, apresentam maior propensão a diminuição da sobrevida.

Os estudos avaliados demonstram ainda a interrelação entre mortalidade e sobrevida com as diversas regiões do País, onde há grandes mudança de acordo com a região analisada e o Município, pois locais em que há menor renda, maiores são os indicadores de morbimortalidade, pela vulnerabilidade das mulheres em virtude dos serviços de saúde prestados, encontrando-se associado a oferta de diagnóstico precoce, tratamento, ações de prevenção inadequadas.

\section{REFERÊNCIAS}

1. Pollock RE, Doroshow JH, Khayat D. Manual de oncologia clínica da UICC. 8.ed. São Paulo: Fundação oncocentro, 2006, 537-56.

2. BRASIL. Estimativa 2012: incidência de câncer no Brasil. Rio de Janeiro: INCA; 2012. Disponivel em: http://www.inca.gov.br.

3. _. . _ _ _._. Controle dos cânceres do colo do útero e da mama. Caderno de Atenção Básica. n.13. Série A. Normas e Manuais Técnicos. Brasília, 2006.

4. Rosa MI, Medeiros LR, Rosa DD, Bozzeti MC, Silva FR, Silva BR. Papilomavírus humano e neoplasia cervical. Cad. Saúde Pública. 2009; 25(5).

5. Mendes KDS, Silveira RCCP, Galvão CM. Revisão integrativa: método de pesquisa para a incorporação de evidencias na saúde e na enfermagem. Texto Contexto Enferm. 2008; 17(4):758-64,

6. Sousa MT, Silva MD, Carvalho R. Revisão integrativa: o que é e como fazer. Rev. Einstein. 2010; 8:102-6,.
7. Castro AA. Avaliação crítica da literatura: roteiro para sessão de artigos de revista. Maceió: AAC; 2003.

8. Basilio DV, Mattos IE. Câncer em mulheres idosas das regiões Sul e Sudeste do Brasil: Evolução da mortalidade no período 1980 - 2005. Rev. Bras. Epidemiol. 2008; 11(2):204-14, .

9. Nakagawa JT, Espinosa MM, Barbieri M, Schirmer J. Carcinoma do colo do útero: taxa de sobrevida e fatores prognósticos em mulheres no Estado de Mato Grosso. Acta Paul. Enferm. 2011; 24(5):631-37.

10. Carmo CC, Luiz RR. Sobrevivência de uma coorte de mulheres com câncer de colo uterino diagnosticadas em um centro de câncer brasileiro. Rev. Saúde Pública. 2011; 45(4).

11. Botero MA, Cervantes CAD, Becerra SCA. Diferenças socioeconômicas nos anos de vida perdidos por câncer de mama e câncer cervical na Colômbia, 1997 e 2007. Rev. Gerenc. Polit. Saúde. 2011; 10(20). 


\section{REFERÊNCIAS}

12. Gamarra CJ, Valente JG, Silva GA. Magnitude da mortalidade por câncer do colo do útero na Região Nordeste do Brasil e fatores socioeconômicos. Rev. Panam. Salud Publica. 2010; 28(2).

13. Meira KC, Silva GA, Silva CMFP, Valente JG. Efeito idade-período-coorte na mortalidade por câncer do colo uterino. Rev. Saúde Pública. 2013; 47(2):274-82.

14. Gonzaga CMR, Freitas Junior R, Barbaresco AA, Martins E, Bernardes BT, Resende APM. Tendência da mortalidade por câncer do colo do útero no Brasil: 1980 a 2009. Cad. Saúde Pública. 2013; 29(3):599-608,.

15. Barbosa IR, Souza DLB, Bernal MM. Costa ICC. Desigualdades regionais na mortalidade por câncer de colo de útero no Brasil: tendências e projeções até o ano 2030. Ciência \& Saúde Coletiva. 2016; 21(1):253-62.

16. Santos RS, Melo ECP. Mortalidade e assistência oncológica no rio de janeiro: câncer de mama e colo uterino. Esc. Anna Nery. 2011; 15(2):410-16.

17. Silva GA, Girianelli VR, Gamarra CJ. Bustamante-Teixeira MT. Tendências de mortalidade por câncer do colo do útero no Brasil, 1981-2006. Cad. Saúde Pública. 2010; 26(12).

18. Neves KC, Silva CA, Ribeiro WA, Fassarela BPA, Alves ALN, Maia ACMSB, Azevedo AL. Adversidades encontradas pelo enfermeiro para a realização da prevenção do câncer do colo de útero. Rev. Saúde coletiva. 2019; 9(49)

19. Zeferino LC. O desafio de reduzir a mortalidade por câncer do colo do útero. Rev. Bras. Ginecol. Obstet. 2008; 30(5):213-5.

20. Alves CMM, Guerra MR, Bastos RR. Tendência de mortalidade por câncer de colo de útero para o Estado de Minas Gerais, Brasil, 19802005. Cad. Saúde Pública, 2009; 25(8):1693-1700,. 